\title{
SOME RECENT ADVANCES IN THE TREATMENT OF FRACTURES.
}

\author{
By H. A. T. FAIRBANK, D.S.O., O.B.E., F.R.C.S. \\ (Consulting Orthopadic Surgeon, King's College Hospital.)
}

When invited to write an introduction to this special number of the PostGraduate Medical Journal one's thoughts naturally turned to the history of Orthopædic Surgery, but this has already formed the subject-in whole or in part - of many addresses and lectures. Our remarks will be confined, therefore, to the more recent history of Orthopædics in this country, with special reference to fracture work.

Before the Great War there were few surgeons who devoted special attention to the affections which were then included in the limited field of Orthopædics, and very few indeed whose practice was confined entirely to the wider field now generally recognized as Orthopædic Surgery. So far as this country was concerned, the War altered all this. It would be more correct, perhaps, to say that Sir Robert Jones and the War altered all this. It was entirely due to his reputation and his leadership that the value of men specializing in this branch of surgery was recognized by the Military authorities, and that the segregation of special cases was started. Since the War orthopædic surgery in this country has come into its own. No general hospital of any size is now considered complete without its Orthopædic Department. The question of the treatment of fractures in the larger hospitals is still a matter of difference of opinion. Before long, however, it is safe to assume that every hospital will have its Fracture Clinic, and-it is to be hoped-beds set apart for fracture cases.

It has never been urged by those anxious to see special organization for the treatment of fracture cases that each fracture clinic should necessarily be in the care of an orthopædic surgeon. All that was asked for was that the cases should be segregated and placed under a surgeon who was willing and anxious to devote time and energy in organizing the work and improving the routine treatment of fractures in his hospital. It seems more than likely, however, that eventually all fracture clinics will be under the care of the orthopædic surgeon of the hospital. There are many arguments in favour of combining the two departments under one head, provided he is given a sufficient number of special assistants. No one who has watched the work in a fracture clinic can doubt the enormous advantage of such segregation to the patients, to the casualty officers, and, in a teaching hospital, to the students. The standard of the routine treatment of the ordinary-fractures has risen enormously, while the more difficult cases are brought to the notice of the surgeon in charge without the unnecessary, and sometimes fatal, delay which was so common before the days of the fracture clinic. Also it seems to be fair to assume that the segregation of fractures is responsible for many of the great advances that have taken place in the treatment of fractures since the War, and particularly during the last ten years or so.

Let us consider some of these advances? To begin with, the pendulum, which had swung too far towards rather inefficient splintage and the early use of massage- "mobilization and massage" as it was sometimes termed-has returned to the older method of uninterrupted immobilization. But there are certain all-important differences between the old and the new methods: immobilization is now much more efficient and this is combined with functional use of the damaged part so far as this is possible without risk of shifting the fragments. The last is made possible by the extensive use of plaster of Paris splints with little or no padding between skin and plaster. Many surgeons, following 
Böhler of Vienna, to whose work in the fracture field the world owes so much, apply the plaster bandages direct to the skin. Others-and the writer is one of them-prefer to cover the limb with thin stockinette and to pad with a little wool a few, but only a few, points where pressure sores are liable to occur, e.g. such a point as the back of the heel. Naturally the use of plaster in this way requires considerable experience if disasters are to be avoided. The advantage to the patient is enormous since, his fracture being really held, he suffers a minimum of pain, while for the same reason he can use the limb surprisingly soon, even walk within a few days on a broken ankle. When the time comes to discard the splintage, the limb has been in use for weeks and is ready to carry on its normal function; the joints are not unduly stiff (even those included in the plaster); the tendency for the limb to swell is much less, and massage, if required at all, is necessary only for a relatively short period. Such a line of treatment must, of course, be followed with discretion and with due regard to the facts in each individual case. For instance, in an ordinary first degree Pott's fracture in which the weight-bearing portion of the tibial articular surface is unbroken, a close fitting plaster enables the patient to walk early without fear of displacement of the foot. It is, of course, essential that the astragalus, if originally displaced, has been accurately restored to its normal position. In a fracture involving the shafts of both tibia and fibula the nature of the fractures and the accuracy of the setting determine the time that should elapse before weight-bearing is permitted. A transverse fracture with the fragments in reasonably good apposition and in perfect alignment can safely bear weight relatively early, while an oblique fracture, particularly if the fragments are in rather poor position, calls for the use of crutches for several weeks.

The importance of the alignment, even in a transverse fracture, is rather apt to be forgotten. In a fracture in the lower half of the tibial shaft, for instance, it is not uncommon to see backward bowing after setting. As union is proverbially slow in this part of the bone, the stimulus which accompanies functional use of the limb is most desirable at the earliest moment compatible with safety, yet in the presence of bowing weight-bearing means a bending strain at the fracture and union may be retarded rather than hastened. There is no doubt about the truth of the dictum, as Lane always insisted, accurate anatomical replacement of the fragments, though not essential in every case, is always highly desirable as it makes recovery of the soft parts easy and is often of the greatest importance in other respects. The more perfect our setting the more likely are we to get a perfect functional result.

This brings to mind another innovation which enjoys an increasing popularity. I refer to skeletal traction, i.e., traction direct to bone distal to the fracture. The temporary use of skeletal traction as an aid to the setting of a fracture, may enable the surgeon to overcome displacement of the fragments in a way which would be quite impossible by any other means except open operation. The pin-or pins, when a second is inserted above the fracture for counter-traction purposes--may be incorporated in the plaster. If so it would seem to be a mistake to leave them for more than a few days, or at most a week or two. More popular is the use of skeletal traction as a method of applying continuous extension for a fractured femur, for instance. The method is painless and the amount of force that can be applied is almost unlimited. It must be emphasized, however, that the method has its disadvantages and risks. It requires constant skilled attention, with repeated X-ray examinations and even then it is not uncommon to see overextension with resulting delay in union or even definite non-union. This risk of non-union is a fact, and a very important fact. Though skeletal traction in skilled 
hands is invaluable in certain cases, e.g., for a compound fracture of both bones in the lower third of the leg, it should not be regarded as the routine method to be used by all for applying extension.

Another fracture in the treatment of which a big advance has been made recently is the crush fracture of a vertebral body. Watson-Jones of Liverpool has shown us that it is possible, particularly when the fracture is in the lower dorsal or lumbar region, where most of these fractures occur, to restore the crushed body to something very near its normal shape by simple hyperextension of the spine. Doped, but not anæsthetized, the patient rests, face downwards, on two tables, his trunk sinking into hyperextension between the two. A plaster corset, reaching both high and low in front, is applied in this position. The principles mentioned above with regard to the functional use of the damaged part are applied in this case, and the patient is encouraged to get up and about within a week or two of the accident. From the first he is made to exercise the spinal muscles, by hyperextending the head and the hip joints in the prone position.

Another fracture we must refer to is that of the neck of the femur. It is no exaggeration to say that the introduction of Smith-Petersen's three-flange nail has revolutionized the treatment of these cases and greatly improved the outlook from every point of view. The advantage over the plaster spica, particularly in the aged, is enormous. Nursing is easy, since there is no plaster surrounding the hip and abdomen. The patient can be propped up at once, gentle movement of the hip joint being painless. The actual technique employed varies with the surgeon, but the vast majority use the modified nail with a central canal, which allows a wire or pin to be inserted first as a guide and its position proved by X-ray films taken in two planes at right angles. No appreciable harm is done if the surgeon is at fault with regard to the direction of his guide. There is no appreciable shock, but it does mean rather a long anæsthetic-few surgeons care for local anæsthesia for these cases-since at least two sets of X-ray films have to be taken, developed and inspected before the nail is actually inserted. Few surgeons now expose the actual fracture: the majority prefer the blind method, the only incision being one on the outer side of the thigh. If the carpentering is done with fair accuracy the chances of getting bony union are much improved, and this with a minimum of discomfort to the patient. The practice of getting patients out of bed, and even bearing weight on the leg within a week or two is against all the rules which experience has shown to be necessary in the conduct of fractures elsewhere in the body treated by operative fixation, and the wisdom of following this course is open to grave doubt. The condition has afforded a great opportunity for original work to the younger surgeons. The brilliant way in which they have risen to the task and are tackling these cases, still rightly regarded as difficult, a glance at recent papers reveals. It bodes well for the future of orthopædic surgery in this country.

With regard to the upper limb little need be said. Plaster is now used as a routine for fractures of the forearm. Only the inexperienced fail to include the elbow and wrist joints. While the actual method of splintage preferred for the average Colles, provided the latter is properly set and the former used efficiently, probably matters little and is best left to the individual preference of the surgeon in charge, but for a badly comminuted Colles, which must be put up with the wrist flexed and the hand deviated to the ulnar side, plaster is far and away the best thing to use.

Fractured scaphoids, often missed or neglected, in the past rarely showed bony union. Nowadays with efficient treatment in plaster for several weeks, with the wrist dorsiflexed, the majority unite. 